\title{
EL PAPEL DE LA GESTIÓN DE CENTROS EDUCATIVOS \\ EN UN MODELO DE APRENDIZAJE \\ BASADO EN COMPETENCIAS
}

\section{The role of management of educational institutions in competency- based learning}

ANALÍA GIMÉNEZ GIUBBANI*

Recibido: 26-03-2016

Revisado: 11-04-2016

Aceptado: 30-04-2016

Resumen. Esta investigación se estructura en dos grandes apartados: en primer lugar, se caracteriza el modelo de aprendizaje basado en competencias y, luego, se analiza la gestión educativa estratégica como alternativa a la gestión tradicional, con énfasis en la competencia de liderazgo. Se tratarán de establecer líneas de sinergia entre este modelo educativo y la gestión de centros; es decir, se presenta el enfoque de las competencias como modelo de enseñanza-aprendizaje y como aporte que ilumina a la gestión educativa.

Palabras clave: aprendizaje basado en competencias, gestión educativa estratégica, liderazgo pedagógico y distribuido.

\footnotetext{
Abstract. This paper is structured in two main sections: the first describes competency-based learning, and the second analyses strategic educational

* Profesora de Filosofía y Licenciada en Humanidades opción Filosofía, por la Universidad de Montevideo. Máster en Educación por la Universidad de Jaén. Doctorado en Filosofía en curso en la Universidad Católica Argentina. Responsable del área de Antropología del Departamento de Formación Humanística de la Universidad Católica del Uruguay. Docente de Antropología y Empresa en dicha Universidad. Docente en educación secundaria y universitaria.
} 
management as an alternative to traditional management, with an emphasis on leadership competency. This paper will aim to establish a link between this educational model and the management of educational institutions; that is to say, the competency-based approach will be presented as a teaching-learning model whilst contributing to educational management.

Keywords: competency-based learning, strategic educational management, pedagogical and distributed leadership. 


\section{INTRODUCCIÓN ${ }^{1}$}

La educación enfrenta en la actualidad muchos cambios y retos. Este apartado se propone resumir, a modo de introducción, algunos de esos desafíos que son producto de cambios de paradigma a nivel interno, pero que tienen su fundamento en transformaciones que se han producido a nivel social. Estas nuevas demandas provienen en parte de la actual sociedad de la información y del conocimiento. En forma cada vez más rápida se acumulan conocimientos en todas las áreas, al tiempo que se difunden con gran rapidez a través de los nuevos medios de comunicación, y se traducen en aplicaciones tecnológicas que modifican nuestra vida.

Este fenómeno tiene varias consecuencias para el sistema educativo. Entre ellas, cada vez más se tiende a pensar los currículos en términos de competencias y capacidades que los alumnos deben desarrollar, una de las cuales es justamente la capacidad para buscar y procesar información.

Frente al cambio constante como sello distintivo de nuestra era, es el conjunto de los elementos que constituyen el sistema educativo lo que se muestra obsoleto. A grandes rasgos, podemos decir que frente a los posicionamientos didácticos clásicos centrados en el aula y en la actividad del profesor, hoy se propugna una enseñanza centrada sobre la actividad autónoma del alumno, con propuestas curriculares más adaptadas a los requerimientos de la vida productiva y comunitaria.

Dos grandes desafíos son comunes a todas las organizaciones humanas en el presente. Por un lado, redefinir su misión, construir una nueva visión que oriente su labor y, simultáneamente, buscar nuevas formas de organización, romper las viejas estructuras por otras más flexibles que permitan adaptarse a entornos cambiantes.

Los sistemas educativos no escapan a esta situación general. En este contexto es preciso volver a pensar hacia dónde deben ir y cómo deben organizarse y conducirse las escuelas; es decir, revisar tanto los objetivos que buscan cumplir en la sociedad, como sus modos de organizarse y funcionar para plasmarlos. A este cambio cultural debemos dar respuesta desde la educación, pero teniendo en cuenta que la organización y la gestión también deben formar parte de esta transformación, tanto a nivel del gobierno de la educación como de los centros educativos.

\footnotetext{
${ }^{1}$ Este artículo es fruto del trabajo de memoria de investigación del máster en Educación realizado entre 2012-2015 (Universidad de Jaén, España).
} 
De algún modo nos encontramos a mitad de camino en este desafío, porque hemos construido nuevas visiones, pero nos cuesta mucho romper las viejas estructuras. Hoy nadie duda, por ejemplo, que la educación deba estar centrada en el alumno más que en el docente, sin embargo las prácticas educativas no acompañan esta convicción; en general, seguimos repitiendo métodos y formas de organización tradicionales.

En este escenario de cambio, la gestión de centros enfrenta varios desafíos que le implican ciertas transformaciones. Uno de ellos podría denominarse "Currículo y transformación"; es decir, los nuevos modelos de enseñanzaaprendizaje tienen dentro de sus objetivos la innovación curricular y es preciso que la gestión educativa asuma conscientemente estas nuevas demandas.

De modo que al presente estudio lo atraviesa una certeza: hacer una opción por un modelo educativo exige una gestión específica y un alto nivel de competencia profesional por parte de los directivos. Este es el fundamento y pertinencia de este trabajo que pretende, a través de una investigación bibliográfica, presentar un modelo de aprendizaje atento a estos desafíos y examinar qué tipo de gestión educativa requiere. En el fondo la pregunta es: ¿qué tipo de gestión impacta positivamente en los aprendizajes?

Esta investigación se estructura en dos grandes apartados: en primer lugar, se caracteriza el modelo de aprendizaje basado en competencias y, luego, se analiza la gestión educativa estratégica como alternativa a la gestión tradicional, con énfasis en la competencia de liderazgo. Se tratarán de establecer líneas de sinergia entre este modelo educativo y la gestión de centros; es decir, se presenta el enfoque de las competencias como modelo de enseñanza-aprendizaje, y como aporte que ilumina a la gestión educativa.

Algunas preguntas, que orientan esta investigación, son:

¿Cuáles son los objetivos y la importancia de un modelo de aprendizaje basado en competencias?

¿Cuál es el papel de la gestión/dirección educativa en este modelo? ¿Cómo debe ser dicha gestión/dirección para el éxito del modelo de aprendizaje basado en competencias?

¿Cuáles son las competencias esenciales del directivo para la implementación de este enfoque? 
¿Qué prácticas favorecen la construcción del liderazgo? ¿Cómo influye este en el mejoramiento de la calidad educativa?

\section{EL MODELO DE APRENDIZAJE BASADO EN COMPETENCIAS}

La expresión competencias se encuentra ampliamente extendida en el mundo de la educación, aunque este concepto es entendido de diferentes maneras y se le asignan significados diversos, no siempre coincidentes. Existen, no obstante, amplios consensos que han permitido desarrollar programas formativos basados en la convergencia entre países, como por ejemplo el proyecto Tuning ${ }^{2}$.

En la siguiente tabla, se incluyen resumidamente los principios pedagógicos que subyacen al enfoque basado en las competencias:

\section{Tabla 1. Principios pedagógicos del modelo basado en competencias}

1. La pretensión de la escuela no es transmitir informaciones sino provocar el desarrollo de competencias básicas.

2. El objetivo de la enseñanza será que los alumnos reconstruyan sus modelos mentales vulgares, sus esquemas de pensamiento.

3. Provocar aprendizaje relevante requiere implicar activamente al estudiante en procesos de búsqueda, estudio, experimentación, reflexión, aplicación y comunicación del conocimiento.

4. El desarrollo de las competencias requiere focalizar en las situaciones reales y proponer actividades auténticas. Buscar anclajes con la vida cotidiana.

5. La organización espacial y temporal de los contextos escolares ha de contemplar la flexibilidad y creatividad requerida por la naturaleza de las tareas auténticas y por las exigencias de vinculación con el entorno social.

6. Aprender en situaciones de incertidumbre y en procesos permanentes de cambio, es condición para aprender a aprender.

7. Preparación de entornos de aprendizaje caracterizados por el intercambio y vivencia de la cultura más viva y elaborada.

8. Estimular la metacognición de cada estudiante, su capacidad para comprender y gobernar su propio proceso de aprender.

9. Favorecer la cooperación entre iguales. Esta incluye diálogo, debate y discrepancia, respeto a las diferencias, saber escuchar, enriquecerse con los demás y tener generosidad para ofrecer lo mejor de sí mismo.

10. Proporcionar entorno cálido, seguro y libre.

\footnotetext{
${ }^{2}$ Para saber más sobre este proyecto, consulte en http://unideusto.org/tuning/
} 


\begin{tabular}{|ll|}
\hline 11. & La evaluación ha de entenderse como formativa. \\
\hline 12. & La función del docente es la de tutorización del aprendizaje. \\
\hline
\end{tabular}

Fuente: elaboración propia a partir de Pérez Gómez (2007, p. 23).

Es interesante señalar dos cuestiones que se desprenden de estos principios y que entendemos centrales para la educación hoy en día. Primero, que la escuela pueda incentivar a los alumnos a desnaturalizar sus esquemas de pensamiento, sus creencias y representaciones básicas, para conducirse con autonomía y ser protagonistas de la construcción del conocimiento. Desnaturalizar significa poner en cuestión, problematizar la realidad. En segundo lugar, que la educación debe promover la capacidad de dialogar en la discrepancia, que es justamente la democracia en sentido amplio.

Las diversas definiciones del concepto de competencia responden a diferentes fuentes, perspectivas y epistemologías. En algunos casos estas posiciones señalan más los componentes operativos que los conceptuales.

Entre estas definiciones operativas se tiene la que el Departamento de Educación de Estados Unidos propuso en el año 2001 como marco para el desarrollo de un sistema nacional de estándares, de evaluación y de certificación de habilidades y destrezas. Allí se señala que una competencia es una combinación entre destrezas, habilidades y conocimientos necesarios para desempeñar una tarea específica (U.S. Department of Education. National Center for Education Statistics, 2002).

En el siguiente cuadro se incluyen otras definiciones de competencia que completan y enriquecen la cuestión:

Tabla 2. Definiciones de competencia

\begin{tabular}{|l|l|}
\hline \multirow{2}{*}{ Lasnier (2000) } & $\begin{array}{l}\text { Un saber hacer complejo, resultado de la } \\
\text { integración, la movilización y la adecuación } \\
\text { de capacidades (conocimientos, actitudes y } \\
\text { habilidades) utilizados eficazmente en } \\
\text { situaciones que tengan un carácter común. }\end{array}$ \\
\hline Proyecto Tuning (2004-2007) & $\begin{array}{l}\text { Combinación dinámica de conocimiento, } \\
\text { comprensión, capacidades y habilidades. }\end{array}$ \\
\hline Perrenoud (2004) & $\begin{array}{l}\text { Aptitud para enfrentar eficazmente una familia } \\
\text { de situaciones análogas, movilizando a }\end{array}$ \\
\hline
\end{tabular}




\begin{tabular}{|l|l|}
\hline & $\begin{array}{l}\text { conciencia y de manera a la vez rápida, } \\
\text { pertinente y creativa, múltiples recursos } \\
\text { cognitivos: saberes, capacidades, micro- } \\
\text { competencias, informaciones, valores, } \\
\text { actitudes, esquemas de percepción, de } \\
\text { evaluación y de razonamiento. }\end{array}$ \\
\hline Rué y Martínez (2005) & $\begin{array}{l}\text { Las tres grandes dimensiones que configuran } \\
\text { una competencia cualquiera son: saber } \\
\text { (conocimientos), saber hacer (habilidades) y } \\
\text { ser (actitudes). }\end{array}$ \\
\hline OCDE - Santillana (2005) & $\begin{array}{l}\text { La capacidad de los alumnos para aplicar } \\
\text { conocimientos y habilidades, y para analizar, } \\
\text { razonar y comunicarse con eficacia cuando } \\
\text { plantean, resuelven e interpretan problemas } \\
\text { relacionados con distintas situaciones. }\end{array}$ \\
\hline Gairín Sallán, J. (2009) & $\begin{array}{l}\text { Combinación de habilidades prácticas, } \\
\text { conocimientos, motivación, valores éticos, } \\
\text { actitudes, emociones y otros componentes } \\
\text { sociales y de comportamientos que se } \\
\text { movilizan conjuntamente para lograr una } \\
\text { acción eficaz. }\end{array}$ \\
\hline $\begin{array}{l}\text { Conjunto de conocimientos, habilidades y } \\
\text { actitudes que se tienen que integrar para } \\
\text { hacer una tarea específica. }\end{array}$ \\
\hline $\begin{array}{l}\text { Actuaciones integrales para identificar, } \\
\text { interpretar, argumentar y resolver problemas } \\
\text { con idoneidad y compromiso ético, } \\
\text { movilizando los diferentes saberes: ser, hacer } \\
\text { yonocer. }\end{array}$ \\
\hline (2010)
\end{tabular}

Fuente: elaboración propia a partir de Gairín Sallán, J. (2009) y otros autores aquí citados.

Efectivamente, revisando la literatura acerca de esta temática, uno de los primeros aspectos que sobresale es la multiplicidad de definiciones que existen de competencia. Al repasar el contenido de estas, importa sintetizar y comentar algunas particularidades:

a. Aunque el conocimiento es un elemento clave de la competencia, no es suficiente para ser competente. En definiciones más explicativas aparecen 
también las capacidades, destrezas, motivaciones, actitudes. Vemos que las competencias tienen un carácter holístico e integrado.

b. Formar personas y profesionales mediante una orientación por competencias implica además que esos conocimientos sean puestos en práctica efectiva en el mundo real. Entonces, al plantearnos la enseñanza de competencias, lo que estamos intentando es facilitar la capacidad de transferir unos aprendizajes, que generalmente se han presentado descontextualizados, a situaciones cercanas a la realidad. Aquí apreciamos el carácter contextual de las competencias y el carácter creativo de la transferencia.

c. La competencia no es algo directamente observable, sino que se infiere a partir de la interpretación de desempeños; se relaciona con una capacidad en acción para responder a situaciones cambiantes. Las competencias suponen un proceso permanente de reflexión para armonizar intenciones con las posibilidades de cada contexto.

d. La competencia supone un saber (conceptual), un saber hacer (procedimental) y un saber ser (actitudinal), acorde con lo que también se señala en el informe de Delors (1995, p. 34). Podemos observar aquí la dimensión ética de las competencias, que se nutren de actitudes, valores y compromisos que las personas van adoptando.

e. Tienen un carácter evolutivo. Se desarrollan, perfeccionan, amplían, o se deterioran y restringen a lo largo de la vida.

El fin de la educación, y de la educación basada en competencias, es el pleno desarrollo del ser humano. Esto supone evidenciar, como lo hacen los autores, que la competencia trasciende el terreno profesional y afecta a la vida de la persona. Asumiendo esta premisa, cabe plantear competencias de corte específico y también genérico, contemplando así el ámbito social, interpersonal, personal y profesional; un acento en la hoy tan aludida integralidad de la educación.

Es así que considerando las posibles clasificaciones de las competencias, se expone una que intenta ser abarcadora, siguiendo la Guía para la evaluación de competencias en el área de ciencias sociales (Gairín Sellán, op. cit., p. 18), que las divide en:

- Competencias específicas, que son propias de un ámbito o titulación y están orientadas a la consecución de un perfil específico del graduado. 
Estas son particulares y precisas a un área o contenido propio y se traducen en la resolución de tareas complejas.

- Competencias genéricas, que son comunes a la mayoría de las titulaciones. Dentro de este bloque encontramos competencias personales como la gestión del tiempo y la responsabilidad del mismo aprendizaje; competencias interpersonales, como comunicarse, trabajar en equipo, liderar o negociar. Estas competencias son transversales a todo el proceso formativo, integrando de manera uniforme el currículo de una carrera en relación a las grandes líneas que marcan el perfil de una institución.

\section{Cambios que conlleva este modelo}

En cierto modo podríamos entender que el término competencia nace como una respuesta a las limitaciones de la enseñanza tradicional. Mas justamente, "la dificultad en la enseñanza de las competencias viene dada (...) porque la forma de enseñarlas implica actividades muy alejadas de la tradición escolar" (Zabala y Arnua, 2007, p. 45). Según los autores, nuestra tradición, basada en la transmisión verbal y reproducción literal de lo aprendido, no alienta la implementación de un modelo de aprendizaje basado en competencias. Este demanda significatividad en los aprendizajes, exige tener que partir de los conocimientos previos de los alumnos, tener en cuenta las motivaciones personales, ofrecer retos y ayudas según las posibilidades reales de cada uno.

Asimismo, cabe subrayar que las competencias tienen un carácter procedimental y funcional, se aprende haciendo, y se enseña para la complejidad. Sin embargo, la escuela heredada está basada en el saber y en el poder del profesor, y no en el saber hacer por parte del alumno; se valora más la capacidad de reproducir que la de aplicar.

Es preciso redefinir el papel del alumno y del profesor en el marco de este enfoque educativo. El alumnado aparece como gestor de su propio aprendizaje y no como mero depositario del mismo. El propósito es que ellos:

- Sean capaces de buscar, seleccionar y tratar la información recibida para crear conocimiento y aplicarlo autónomamente.

- Aprendan a afrontar las incertidumbres propias del conocimiento y asuman que la solución de unos problemas genera otros. 
- Aprendan a convivir, participando activamente en un mundo globalizado, interrelacionado y cambiante.

- Adquieran una formación ética, que se obtiene, más allá de los contenidos de una materia, mediante un ejercicio constante de reflexión y práctica democrática. (Grupo de Competencias básicas de la Consejería de Educación de Cantabria, 2007, pp. 13 y 14)

Por su parte, el docente se transforma en un facilitador de este proceso que busca la autonomía del alumno. Debe potenciar la construcción del conocimiento, la reflexión crítica y el uso o aplicación de los saberes adquiridos, siempre en contextos significativos para que los aprendizajes adquieran sentido. Este nuevo perfil ha de incidir en la función del docente, que de la mera transmisión debe pasar a provocar la reconstrucción del conocimiento desde la experiencia del alumno (ibídem).

Es fundamental interrogarse si el maestro se erige como un simple poseedor y transmisor de conocimientos; o si por el contrario asume el rol de mediador y guía en la construcción del saber y en el pleno desarrollo de la individualidad de sus alumnos. Ambas maneras de entender dicha tarea implican distintas consecuencias y suponen una concepción previa de la verdad. En el primer caso esta es entendida como realidad preexistente y el alumno como depósito de esa verdad a transmitir; mientras que en el segundo caso, la verdad es concebida como construcción en común y proceso hacia un saber inagotable, fruto de la contingencia y el devenir históricos, pero siempre de acuerdo a la experiencia humana vital.

En esta segunda línea se coloca el modelo de las competencias como vimos. No es rol de quien enseña ofrecer la realidad de manera acabada, ni convertirse en sujeto de una exploración ajena, sino acompañar el camino de autoconocimiento de cada uno de sus alumnos.

Del mismo modo, el marco de un enfoque educativo por competencias requiere una visión de los objetivos, contenidos y criterios de evaluación, en armonía. Los objetivos deben ser interpretados y desarrollados en términos de competencia, estas son las verdaderas finalidades del proceso educativo. Asimismo, los contenidos deben organizarse y jerarquizarse para contribuir al desarrollo y adquisición de las competencias. Aquí el profesorado tiene un rol clave; ya no debo preguntarme qué voy a dar en primer lugar. Y por lo que respecta a los criterios de evaluación, estos deben jugar el papel de nexo de interrelación entre las 
competencias que se definen, los objetivos que se persiguen y los contenidos que se han planificado. ${ }^{3}$

La decisión sobre una enseñanza basada en competencias plantea cambios también en lo que refiere a la gestión y organización de los centros educativos. Resultan muy pertinentes las siguientes preguntas:

¿Puede el sistema educativo hacerse cargo de estos nuevos desafíos con sus actuales estructuras de organización y funcionamiento? ¿Son aptas las modalidades en que los sistemas educativos se organizaron a lo largo de un siglo para responder adecuadamente a los cambios en la sociedad del presente? ¿Qué es necesario cambiar? (UNESCO-IIPE-BA, 2000, módulo 1, p. 19)

En casi todos los países ha sido muy difícil llegar a la toma de conciencia de que las competencias necesarias para dirigir una escuela no son las mismas que las que se requieren por ejemplo, para ser profesor. Esto, que pudo haber sido válido en otros momentos, resulta hoy insuficiente. Cada vez más es indispensable reconcebir los aspectos esenciales de la gestión de los centros educativos, que no son indiferentes sino facilitadores o condicionantes del cumplimiento de los objetivos de la educación. A esta concientización todavía le queda camino por recorrer y profundizar.

La gestión educativa ha sido tradicionalmente un aspecto secundario en la escuela, centrada en sus procesos administrativos y no profesionalizada. Este problema se debe en parte a que la gestión ha estado ligada estrictamente a lo empresarial y separada de lo educativo. Pero si bien el aula es el espacio privilegiado de la educación, es en la institución como unidad de sentido donde se juega la calidad educativa.

Hoy hablamos de autonomía institucional pero cuando los centros se encuentran frente a la toma de decisiones, chocan con los modelos de organización y estilos de gestión vigentes. Es necesaria una revisión, pero ¿cómo tendrían que ser las escuelas para llevarla a cabo?

\footnotetext{
${ }^{3}$ En este punto, es importante tener en cuenta la necesidad de adecuar las herramientas de evaluación a las evaluaciones internacionales ya existentes. Si consideramos la evaluación PISA, el concepto clave es justamente el de competencia (OCDE-Santillana, 2005, p. 23). Este aspecto es interesante para hacer un apunte sobre el caso de Uruguay, cuya participación en 2012 fue la peor desde que asiste a las pruebas (2003). Estos resultados invitan a pensar en qué medida el sistema educacional está entregándole a todos sus alumnos las herramientas para que se desenvuelvan de manera efectiva en el mundo social y económico. El desafío para Uruguay, como para el resto de Latinoamérica, es asegurar que todos los alumnos logren un nivel de competencias básico.
} 
Aguerrondo (1996) es una de las autoras que ha puesto el acento sobre esta problemática y señala dos características interesantes, que resumimos a continuación:

Tabla 3. Los rasgos del nuevo modelo de gestión y organización escolar

\section{Una organización que aprenda}

Una forma de pensar la organización y gestión de centros es desde el paradigma del aprendizaje institucional y no desde el control, que significa por un lado reconocer y corregir el error (como desvío de los objetivos), pero también flexibilizar a la organización facilitando el aprendizaje de nuevos procedimientos y nuevas respuestas frente a los nuevos desafíos.

En suma, una organización flexible y heterodirigida, que no sólo acepta el desafío del entorno sino que es capaz de aprovecharse de él como motor de la transformación institucional.

\section{Una gestión que conduzca}

Cuando se trata de enfrentar cambios, las escuelas tienen problemas de resistencia y falta de flexibilidad. La educación en sí misma y la cultura tiene un ritmo de cambio relativamente lento.

Ante esto, el campo de la gestión es el que tiene que ver con la probabilidad de que una vez tomadas las decisiones, se concreten de manera eficaz, una gestión que muestre resultados y que conduzca hacia los objetivos fijados. El perfil concreto del quehacer de la gestión eficiente se resume en su capacidad de generar y sostener líneas de acción.

Fuente: elaboración propia a partir de Aguerrondo (1996, pp. 26-29).

Esta misma autora plantea dos desafíos para la gestión y la organización de los centros:

- Enfrentar la masividad, que se traduce en la necesidad de profesionalizar la gestión porque la naturaleza de los problemas cambió, los modelos originales se volvieron ineficientes, y entró en crisis la respuesta de un servicio homogéneo.

- Responder por sus resultados, lo que nos enfrenta con la pregunta: ¿con qué organización y estilo de gestión se logran resultados eficaces? (op. cit., pp. 3234)

Cabe resaltar que, aunque nos encontramos a dos décadas de estas reflexiones, las preocupaciones e intenciones continúan siendo las mismas (en líneas generales); lo que nos indica que es preciso traducir y llevar a la acción estos cambios.

\section{EL PAPEL DE LA GESTIÓN EDUCATIVA DESDE Y PARA EL DESARROLLO DE COMPETENCIAS}


"Se tiende a una dirección pedagógica, encaminada al incremento de los aprendizajes y de los resultados del centro escolar" (Bolivar-Botía, 2010, p. 81)

\section{La gestión educativa estratégica}

Para comprender las múltiples acepciones del término gestión, se han propuesto las siguientes expresiones: piloteo, innovación, exploración, mejora continua, profesionalización, identificación de fortalezas y dificultades, pensamiento para la acción, liderazgo pedagógico, visión, comunicación, aprendizaje, construcción de redes, anticipación, decisión, evaluación. Todas tienen que ver de algún modo con la participación, como reconocimiento de que la gestión es una actividad de actores colectivos.

La transformación en la que estamos inmersos nos impone transitar hacia la gestión educativa estratégica como alternativa a la gestión tradicional. A continuación incluimos una tabla con sus características principales:

Tabla 4: Señas de identidad de la gestión educativa estratégica

\begin{tabular}{|l|}
\hline Gestión educativa estratégica \\
\hline Centralidad de lo pedagógico. \\
\hline Habilidades para tratar con lo complejo. \\
\hline Trabajo en equipo. \\
\hline Apertura al aprendizaje y a la innovación. \\
\hline Asesoramiento y orientación profesionales. \\
\hline Culturas organizacionales cohesionadas por una visión de futuro. \\
\hline Pensamiento e intervenciones sistémicas y estratégicas. \\
\hline Reconfiguración, nuevas competencias y profesionalización. \\
\hline Liderazgo pedagógico. \\
\hline Aprendizaje organizacional. \\
\hline
\end{tabular}

Fuente: elaboración propia a partir de UNESCO-IIPE-BA (2000, módulo 2, pp. 15, 19-23, 27-29). 
Algunas reflexiones pueden extraerse del cuadro anterior. En primer lugar, la reconfiguración solo puede encararse si se promueve desde la capacidad de trabajar en equipos, que posibiliten la puesta en común de las experiencias, favoreciendo espacios de intercambio e innovación. De este modo se construye esa organización inteligente y abierta al aprendizaje, que reconoce la necesidad de estos espacios para crecer en participación y responsabilidad colectiva.

En segundo lugar, la gestión educativa puede entenderse como un conjunto de acciones y procesos teórico-prácticos; es un saber de síntesis capaz de ligar conocimiento y acción, tendiendo al mejoramiento continuo de las prácticas educativas. Es una nueva forma de comprender y conducir la organización escolar, que debe reconocer como uno de sus fundamentos el cálculo estratégico situacional, de tal modo que llegue a ser un proceso generador de decisiones específicas. La dirección estratégica es el proceso mediante el cual integramos el pensamiento y el accionar estratégico en la práctica creadora.

En tercer lugar, la gestión educativa tiene que ver con la resolución de conflictos, que se plantean entre lo previsto y lo contingente, y con el abandono de visiones simples para asumir la complejidad e incertidumbre propias de la realidad educativa. Porque la gestión educativa implica y fomenta la integración de diversos saberes, prácticas y competencias.

El estudio de UNESCO-IIPE-BA (2000) sostiene que "todas las actividades de la gestión educativa pueden integrarse en estas tres claves: reflexión, decisión y liderazgo". Asimismo identifica tres componentes inseparables y fundamentales de la gestión educativa estratégica: "pensamiento sistémico y estratégico, liderazgo pedagógico y aprendizaje organizacional" (módulo 2, pp. 27-29).

En este trabajo se hace énfasis en el liderazgo pedagógico y distribuido como competencia indispensable para la gestión en la actualidad. Este acento se fundamenta en que "la agenda próxima, también en los países iberoamericanos, de acuerdo con las orientaciones reflejadas en la literatura internacional, es pasar de una dirección limitada a la gestión administrativa de las escuelas a un liderazgo para el aprendizaje, que vincula su ejercicio con los logros académicos del alumnado y con los resultados del establecimiento educacional" (Bolívar- Botía, 2010, p. 81).

La gestión ha evolucionado desde una dirección por acontecimientos (aseguradora, reactiva, no anticipativa, individualista) a una dirección participativa, sobre la base de la dirección por objetivos y por resultados, y con la influencia del 
liderazgo. Esta dirección supone un perfeccionamiento de los procesos de gestión, presumiendo una activa participación de todos, sustentada en la conducción de equipos que se autodirigen, donde se afianza el liderazgo. Se comienza a diseñar la llamada dirección por valores que llena de sentido, humaniza, y completa a través de valores compartidos, al resto de los tipos de dirección que le antecedieron, sin ignorarlas.

Creemos efectivamente que este es el tipo de gestión educativa que demanda un modelo de aprendizaje basado en competencias. Al mismo tiempo, dicho modelo puede iluminar y orientar las prácticas de gestión en la actualidad. De modo que se constituye una suerte de interrelación entre competencias y gestión. Si queremos educar en competencias, la institución toda debe estar comprometida e insuflada con este enfoque.

\section{Competencias para la gestión educativa estratégica}

El enfoque de las competencias parece ofrecer una respuesta innovadora relacionada con los desafíos de la construcción de una nueva profesionalidad en la educación. Pilotear una organización requiere nuevas capacidades de sus gestores, nuevas competencias que incluyen la capacidad de reflexión, creatividad, decisión, liderazgo y acción.

La realidad dinámica y compleja debe ser reconstruida en modelos descriptivos y explicativos a través de procesos de análisis y síntesis. El gestor debe identificar los distintos patrones que se manifiestan debajo de los diversos fenómenos y casos concretos. Los modelos conceptuales específicos permitirán luego desarrollar intervenciones dirigidas a manipular aquellas variables que están dentro del espacio estratégico de acción del gestor.

Del mismo modo, resulta esencial la capacidad intelectual de comprender los fenómenos como interdependencias constituyentes de un sistema y, además, articulados en cadenas de causalidad recíproca. Desde aquí es posible que el gestor imagine y construya cursos y escenarios alternativos de acción-intervención.

La capacidad de experimentación es relevante a la hora de situar a los gestores en los términos mismos de la intervención, tanto desde un punto de vista profesional como interpersonal. 
Finalmente, una de las competencias principales para los responsables de la gestión educativa es la capacidad para trabajar en equipo. Más aun, resulta fundamental que el gestor sea capaz de educar a otros en el desarrollo de esta capacidad. Esto supone una reducción del aislamiento, que tradicionalmente ha caracterizado al trabajo en la educación, y una apertura a la complementariedad, a la aceptación de los demás.

Por su parte, Medina y Gómez (2014), en su estudio sobre el liderazgo pedagógico, señalan que las competencias más valoradas en los directivos son la gestión humana y la técnica, y subrayan la importancia de la formación del directivo como líder pedagógico.

La competencia de gestión significa claridad y coherencia en la toma de decisiones. Implica que el líder ha de comprometerse con la mejora de la docencia y de las prácticas educativas, como condición para lograr el aprendizaje de los estudiantes.

La competencia de carácter humano tiene un lugar prioritario en la educación actualmente. Demanda de los directivos la combinación de inteligencia emocional, empatía, comunicación, sensibilidad, honestidad y apertura. El liderazgo tiene, para estos autores, una naturaleza eminentemente humanista en tanto exige compromiso con los valores educativos.

La competencia técnica afecta al dominio del diseño y del desarrollo del programa en las instituciones educativas, implicando a expertos que conozcan los elementos más representativos a aplicar, especialmente el significado y el impacto que para la educación de los estudiantes se espera del proyecto de mejora. Esta competencia requiere dominio de los medios TIC, espacios virtuales y otros recursos para contribuir al diseño y ejecución de programas creativos e innovadores de mejora (pp. 94 y 95).

La competencia que aparece como transversal es la de liderazgo, porque el trabajo en equipo no garantiza la gestación de nuevas propuestas si no existe la capacidad de aterrizar las ideas en proyectos realizables. Si no existe clara capacidad de liderazgo, difícilmente la autonomía y el trabajo en equipo den sus frutos.

\section{El liderazgo pedagógico y distribuido}


Un relevante informe de la OCDE (Mejorar el liderazgo escolar) señala que "en muchos países hay una creciente inquietud de que la función de los directores, diseñada para las necesidades de una época diferente, pueda no ser la adecuada para enfrentar los desafíos de liderazgo que las escuelas encaran en el siglo XXI" (Pont, Nusche y Moorman, 2009, vol. 1, p. 27).

Medina y Gómez (2014) indican que:

Los modelos de liderazgo más representativos para promover procesos innovadores en los Centros son: transformacional, de armonía emocional, de colaboración, distribuido, etc., que consideran el liderazgo como una síntesis de múltiples realidades sociorrelacionales, que sitúan en el eje de su identidad las emociones y las necesidades de las personas que forman la organización, actuando como generador del desarrollo integral del resto de las personas de la Institución. (p. 92)

El liderazgo educativo o dirección pedagógica de las escuelas se está constituyendo, en el contexto internacional, en un factor de primer orden en el mejoramiento de la educación. Diversos estudios internacionales así lo indican.

El Informe McKinsey señala que un buen liderazgo escolar es un factor determinante de la calidad de la educación, para lo que se deben seleccionar y formar excelentes directivos (Barber y Mourshed, 2008, pp. 32 y 33).

Según Bolívar-Botía (2010), "la capacidad para el mejoramiento de un establecimiento escolar depende, de manera relevante, de equipos directivos con liderazgo que contribuyan activamente a dinamizar, apoyar y animar su desarrollo, de manera que pueda construir su capacidad interna de mejora" (p. 81).

En sentido general, entendemos como liderazgo escolar "la labor de movilizar e influenciar a otros para articular y lograr las intenciones y metas compartidas de la escuela" (Leithwood, 2009, p. 20).

Según el texto del IIPE, el liderazgo es un conjunto diverso de prácticas pedagógicas e innovadoras, que buscan facilitar, orientar y regular procesos complejos de delegación, cooperación y formación de los docentes, directivos y demás personas que se desempeñan en la educación. Las prácticas de liderazgo activan a las organizaciones educativas para recuperar el sentido y la misión pedagógica, desarrollada a partir de objetivos tendientes a lograr aprendizajes 
significativos para los estudiantes (UNESCO-IIPE-BA, op. cit., módulo 2, pp. 28 y 29).

Lo más relevante del liderazgo es que su potencial se vincula directamente con el aprendizaje profundo; liderazgo pedagógico, "liderazgo centrado en el aprendizaje" (Bolívar-Botía, 2010, p. 83).

La gestión requiere prácticas de liderazgo para concertar, reflexionar, planificar, dirigir, acompañar, comunicar, motivar y educar en la transformación educativa. La construcción de escuelas eficaces requiere líderes pedagógicos que promuevan procesos de aprendizaje y contribuyan a sensibilizar y convocar a trabajar en colaboración. En definitiva, el liderazgo promueve el trabajo generativo. Es la dimensión de la gestión educativa estratégica que asume que los cambios a emprender requieren identificar y plantear los problemas colectivos, evaluarlos en función de los valores a profundizar, para lograr resultados útiles.

Incluimos el siguiente cuadro con algunas acciones que favorecen el liderazgo y, al mismo tiempo, posibilitan y promueven la gestión para el aprendizaje:

Tabla 5: Contribución de las prácticas de liderazgo y acciones que lo favorecen

\begin{tabular}{|l|l|}
\hline Las prácticas de liderazgo son un medio para: & $\begin{array}{l}\text { Prácticas que favorecen la construcción del } \\
\text { liderazgo: }\end{array}$ \\
\hline Generar aprendizaje organizacional y social. & $\begin{array}{l}\text { Inspirar la necesidad de generar } \\
\text { transformaciones. }\end{array}$ \\
\hline Resolver colectivamente problemas nuevos. & Generar una visión de futuro. \\
\hline Redefinir los valores. & Comunicar esa visión de futuro. \\
\hline $\begin{array}{l}\text { Ajustar los procesos de acción para alcanzar } \\
\text { esos valores. }\end{array}$ & Promover el trabajo en equipos. \\
\hline $\begin{array}{l}\text { Estimular el desarrollo de otras formas de } \\
\text { comprender y de actuar. }\end{array}$ & $\begin{array}{l}\text { Brindar orientación que desarrolle el espíritu de } \\
\text { logro. }\end{array}$ \\
\hline $\begin{array}{l}\text { Ampliar los procesos de mejora continua. } \\
\text { profundo. }\end{array}$ & Consolidar los avances en las transformaciones. \\
\hline $\begin{array}{l}\text { Solventar procesos extendidos y continuos de } \\
\text { formación para el fortalecimiento de } \\
\text { competencias complejas, tanto individuales como } \\
\text { colectivas. }\end{array}$ & $\begin{array}{l}\text { Actualizar el aprendizaje y acumular } \\
\text { conocimiento. }\end{array}$ \\
\hline
\end{tabular}

Fuente: elaboración propia a partir de UNESCO-IPPE-BA (2000, módulo 3, pp. 18 y19). 
En el referido programa promovido por la OCDE, la mejora del liderazgo escolar pasa por cuatro grandes líneas de acción: (re)definir las responsabilidades; distribuir el liderazgo escolar; adquirir las habilidades y competencias necesarias para un liderazgo eficaz; y hacer del liderazgo una profesión atractiva. Estos tópicos constituyen los capítulos del citado volumen (Pont, Nusche y Moorman, 2009, vol. 1).

La acción del líder debe situarse asimismo en el perfeccionamiento de las prácticas docentes, para de ese modo contribuir con la mejora de los logros de los alumnos. Esta tarea, que ha sido entendida en muchos países como alejada del ámbito de competencia de la dirección, es fundamental, porque si la labor docente es factor determinante del aprendizaje de los estudiantes, los directores deben crear condiciones para que el docente ejerza mejor su trabajo. ${ }^{4}$

Otro aspecto interesante del ejercicio de la dirección como liderazgo es entenderlo como una práctica distribuida en el conjunto de la organización; se trata de compartir el liderazgo, de generar responsabilidades comunes. Esto supone abogar por un liderazgo distribuido entre todos los miembros de la institución, porque la mejora de la calidad educativa no puede recaer exclusivamente en la dirección.

Como explican López-Yáñez y Lavié-Martínez (2010), "el liderazgo distribuido traslada el ejercicio de la influencia desde la cúspide de la jerarquía organizativa hacia los equipos mismos de trabajo y hacia los docentes" (pp. 72 y 73). En esta línea, Horn y Marfán (2010) subrayan el impacto del liderazgo participativo orientado a estimular la iniciativa del profesorado (p. 91).

Si queremos una organización que funcione, se necesitan más personas que el director; se requiere desarrollar la capacidad de liderazgo en los demás, el compromiso y el trabajo colaborativo. Esto equivale a ampliar la capacidad humana de la institución, no a conceder más comodidad o menos responsabilidad al líder formal. Por el contrario, esto representa más exigencia para él, en tanto debe coordinar y supervisar todo ese liderazgo disperso (López-Yáñez y Manuel LaviéMartínez, op. cit., p. 76). Esta forma de entender y practicar el liderazgo tiende y refuerza lo que recientemente se ha llamado "comunidad profesional de aprendizaje" (Bolívar-Botía, op. cit., p. 92).

\footnotetext{
${ }^{4}$ A título de ejemplo, en Chile, un amplio equipo impulsado por el Centro de Estudios de Políticas y Prácticas en Educación (CEPPE) en unión con la Fundación Chile, está llevando a cabo una investigación sobre liderazgo educativo y resultados de aprendizaje de los alumnos. Disponible en http://www.redalyc.org/pdf/551/55114063003.pdf
} 
"Cuando hay distribución del liderazgo el compromiso de los pares es más influyente que cuando sólo hay un liderazgo administrativo" (Leithwood, op. cit., p. 126). Ciertamente, el liderazgo ejerce una mayor influencia en los aprendizajes y en la mejora educativa cuando está distribuido. No obstante, esto es poco relevante, si no se establecen buenas formas de distribución: qué tareas se distribuyen, niveles de distribución y de acuerdo con qué patrones.

Acerca del liderazgo pedagógico y distribuido han proliferado diversos estudios en la última década. En este trabajo hemos consultado especialmente a Bolívar- Botía (2010) y Medina y Gómez (2014).

Como se puede advertir existe una clara retroalimentación entre la buena gestión de centros y estas prácticas de liderazgo pedagógico y distribuido. Al mismo tiempo, esto repercute en la mejora de la calidad educativa en general y del aprendizaje significativo de los estudiantes en particular.

\section{CONCLUSIONES}

Tal como señalábamos al comienzo, la motivación para realizar este estudio fue la de investigar una realidad educativa que cada vez se presenta como más compleja y cambiante, donde las demandas son heterogéneas, y la tendencia se inclina hacia la necesidad de un mayor énfasis en un aprendizaje significativo y relevante para todos los alumnos.

Una de las conclusiones de este trabajo, es que se hace necesario que la gestión de las instituciones educativas acompañe estos cambios que se producen a nivel social y educacional. Aquí aparecen nuevos modelos de enseñanzaaprendizaje donde se sitúa el basado en competencias.

El propósito entonces, sin ánimo de agotar la cuestión, fue aportar al debate acerca de la mejora de la calidad educativa a través de la innovación y analizar cuál es el papel que cumple la gestión de centros en este proceso que creemos consiste en transformar esquemas y modelos antiguos en educación y gestión. En esta línea, el enfoque de las competencias se mostró como transversal, porque pudimos ver que significa un aporte como modelo de enseñanza al tiempo que como modelo de gestión.

Esto es producto de concebir a las organizaciones como unidades de sentido, y no como compartimentos aislados de alumnos, docentes, directivos, familias. La 
retroalimentación, que a través del modelo de las competencias observamos entre gestión y aprendizaje, se debe a esta obligación de construir comunidades de aprendizaje en torno a los sistemas educativos.

Intentando dar respuesta a los objetivos planteados, podemos decir que la virtud en la actualidad del modelo de aprendizaje basado en competencias, reside fundamentalmente en que contribuye a que los alumnos sean autónomos en sus aprendizajes y asuman que la construcción del saber está en sus manos. El alumno debe pensarse como protagonista de la fundación de valores, saberes y actitudes ante la realidad. Del mismo modo, este modelo es un referente en la convicción de que la educación de las personas debe ser integral.

El enfoque de las competencias además nos demuestra que la verdad no es algo estático, sino que colectivamente participamos en su propio dinamismo. Asimismo, nos sitúa de cara a la construcción de nuestra identidad individual y colectiva. Es finalidad primordial de la educación colocarnos ante esta pregunta que nos hace la historia: ¿quiénes somos?

En lo que refiere a la gestión de centros, hemos logrado dilucidar qué tipo de gestión demanda la implementación de esta modalidad de enseñanza-aprendizaje. Una gestión educativa estratégica que conduzca, en el marco de una organización que aprenda; que incluya pensamiento y acción, tendientes a una práctica creadora y humanizadora de la educación.

Asimismo hemos explorado qué competencias deben poseer los responsables de los centros educativos, agrupadas y resumidas en competencia de gestión, humana y técnica. Nos centramos especialmente en el liderazgo como competencia básica, que es una prioridad política a nivel mundial; un liderazgo pedagógico (abierto al aprendizaje) y distribuido (entre todos los miembros de la comunidad educativa) como clave para dirigir las organizaciones actuales.

Finalmente cabe señalar que si bien esta investigación es de carácter teórico, en una segunda instancia podría traducirse en resultados prácticos, como muestran otros estudios del mismo corte.

\section{REFERENCIAS}

Aguerrondo, I. (1996). La escuela como organización inteligente. Argentina: Troquel. Barber, M. y Mourshed, M. (2008). Cómo hicieron los sistemas educativos con mejor desempeño del mundo para alcanzar sus objetivos. Chile: Informe McKinsey. 
Disponible en http://www.oei.es/pdfs/documento preal41.pdf [Accedido en febrero de 2016].

Bolívar-Botía, A. (2010). ¿Cómo un liderazgo pedagógico y distribuido mejora los logros académicos? Revisión de la investigación y propuesta. Magis. Revista Internacional de Investigación en Educación, 3 (5), pp. 79-106.

Delors, J. (1995). La educación encierra un tesoro. Informe sobre la educación para el siglo XXI. París: UNESCO.

Gairín Sallán, J. (Coord.). (2009). Guía para la evaluación de competencias en el área de ciencias sociales. Barcelona: Agència per a la Qualitat del Sistema Universitari de Catalunya.

Grupo de Competencias básicas de la Consejería de Educación de Cantabria. (2007). Las competencias básicas y el currículo: orientaciones generales. Cuadernos de Educación de Cantabria 2. Consejería de Educación de Cantabria. Disponible en https://www.educantabria.es/docs/info institucional/publicaciones/2007/Cuade rnos Educacion 2.PDF [Accedido en febrero de 2016].

Horn, A. y Marfan, J. (2010). Relación entre liderazgo educativo y desempeño escolar: Revisión de la investigación en Chile. Psicoperspectivas, 9 (2), 82104.

http://www.psicoperspectivas.cl/index.php/psicoperspectivas/article/viewFile/1 16/112 [Accedido en febrero de 2016].

OCDE-Santillana. (2005). Informe PISA 2003. Aprender para el mundo del mañana. Programa para la evaluación internacional de alumnos. OCDE-Santillana. Disponible en http://www.oecd.org/pisa/39732493.pdf. [Accedido en febrero de 2016].

Lasnier, F. (2000) Réussir la formation par compétences. Montreal: Guérin.

Leithwood, K. (2009). ¿Cómo liderar nuestras escuelas? Aportes desde la investigación. Santiago de Chile: Fundación Chile. Disponible en http://www.fch.cl/wp-content/uploads/2012/08/Libro Liethwood.pdf [Accedido en febrero de 2016].

López-Yáñez y Lavié-Martínez. (2010). Liderazgo para sostener procesos de innovación en la escuela. Profesorado, vol. 14, $n^{\circ}$ 1, 71-92. Disponible en http://www.ugr.es/ recfpro/rev141ART4.pdf [Accedido en febrero de 2016]. 
Medina Rivilla, A. y Gómez Díaz, R. (2014). El liderazgo pedagógico: competencias necesarias para desarrollar un programa de mejora en un centro de educación secundaria. Perspectiva Educacional. Formación de Profesores, 53 (1), 91-113.

Pérez Gómez, A.I. (2007). La naturaleza de las competencias básicas y sus aplicaciones pedagógicas. Cuadernos de Educación de Cantabria 1. Consejería de Educación de Cantabria. Disponible en http://www.educantabria.es/docs/info institucional/publicaciones/2007/Cuader nos Educacion 1.PDF?phpMyAdmin=DxoCAdBlc\%2CANuNIkvc-WZcMiFvc [Accedido en febrero de 2016].

Perrenoud, P. (2004). Diez nuevas competencias para enseñar. Barcelona: Graó.

Pont, B., Nusche, D., y Moorman, H. (2009). Mejorar el liderazgo escolar. Vol. 1: política y práctica. OCDE. Traducción y edición: Gilda Moreno y Laura Valencia. Disponible en http://www.oecd.org/edu/school/44374937.pdf [Accedido en febrero de 2016].

Proyecto Tuning. Informe final (2004-2007). Reflexiones y perspectivas de la Educación Superior en América Latina. Disponible en http://tuning.unideusto.org/tuningal/index.php?option=com docman\&ltemid=1 91\&task=view category\&catid=22\&order=dmdate published\&ascdesc=DESC [Accedido en febrero de 2016].

Rué, J. y Martínez, M. (2005). Les titulacions UAB en l'Espai Europeu d’Educació Superior. Cerdanyola del Vallès: IDES-UAB.

Tobón et al. (2010). Secuencias didácticas: aprendizaje y evaluación de competencias en educación superior. México: Pearson.

UNESCO-IIPE-BA. (2000). Desafíos de la educación. Diez módulos destinados a los responsables de los procesos de transformación educativa. Buenos Aires: UNESCO-IIPE-Ministerio de Educación de la Nación. Disponible en http://unesdoc.unesco.org/images/0015/001591/159155s.pdf [Accedido en febrero de 2016].

U.S. Department of Education, National Center for Education Statistics. (2002). Defining and Assessing Learning: Exploring Competency-Based Initiatives. Disponible en http://nces.ed.gov/pubs2002/2002159.pdf [Accedido en febrero de 2016]. 
Zabala, A. y Arnua, L. (2007). La enseñanza de las competencias. Revista Aula de innovación educativa, 161, 40-46. Disponible en http://upvv.clavijero.edu.mx/cursos/ObservacionPracticalll/vector2/tarea6/docu mentos/Zabala Arnua.pdf [Accedido en febrero de 2016]. 